\title{
Calcinosis tumoral de rodilla
}

\section{Tumoral calcinosis of the knee}

\author{
Juan Pablo Restrepo, María del Pilar Molina • Armenia
}

\begin{abstract}
Resumen
La calcinosis tumoral o síndrome de Teutschlander es una condición benigna y rara caracterizada por la presencia de masas calcificadas de ubicación periarticular en la cadera, hombro, codo y menor frecuencia en pies, tobillos y rodillas. Se cree que hay una alteración genética en el GALNT3 y FGF23 que regulan la absorción renal de fosfato. Usualmente esta lesión es asintomática y rara vez puede dar síntomas producto de compresión de estructuras vecinas. La imaginología permite habitualmente realizar el diagnóstico. Hasta el momento no hay tratamiento médico exitoso y se sigue recomendando la escisión quirúrgica aunque hay recurrencias (Acta Med Colomb 2010; 35: 185-186).
\end{abstract}

Palabras clave: calcinosis tumoral, rodilla, artritis reumatoide.

\section{Abstract}

Tumoral calcinosis or Teutschlander syndrome is a rare and benign condition characterized by the presence of calcified masses around the hip, elbow, and less frequently feet, ankles and knees. It is believed that there is a genetic alteration in GALNT3 and FGF23 which regulate the renal absorption of phosphate. Usually it is an asymptomatic lesion and occasionally it produces symptoms of compression to peripheral tissues. Imaging permits the diagnosis. There is no successful medical treatment and surgical excision is recommended although recurrences are frequent (Acta Med Colomb 2010; 35: 185-186).

Key words: tumoral calcinosis, knee, rheumatoid arthritis.
Dr. Juan Pablo Restrepo: Internista, Reumatólogo, Profesor, Universidad del Quindío; Dra. María del Pilar Molina: Médica y Cirujana, Universidad Libre. Armenia, Quindío. Correspondencia: Dr. Juan Pablo Restrepo. Cra 13 No. 1N-35, Consultorio 412. Armenia, Quindío.

E-mail: jprestrepo@1ycos.com

Recibido: 29/X/09 Aceptado: 26/X/10

\section{Introducción}

La calcinosis tumoral es una condición rara y benigna caracterizada por la presencia de masas calcificadas periarticulares usualmente localizadas alrededor de grandes articulaciones. Estas lesiones no afectan la articulación subyacente y rara vez ocasionan síntomas compresivos a estructuras vecinas. A continuación describimos un caso de una mujer de raza negra con calcinosis tumoral en presencia de una artritis reumatoide.

\section{Historia clínica}

Paciente femenina, raza negra de 53 años de edad, con diagnóstico de artritis reumatoide seropositiva de 23 años de evolución, quien venía con mal control de la actividad inflamatoria; subtratada por varios años con fármacos modificadores de la enfermedad (DMARDS) tipo metotrexate y cloroquina de manera irregular. Acude a consulta a nuestro servicio hace dos años por presentar dolor e inflamación en muñecas, metacarpofalángicas, interfalángicas proximales, codos, rodillas acompañado de rigidez matinal mayor de 30 minutos. Hace un año la paciente empezó a referir un dolor de características mecánicas en rodillas con limitación funcional.
El examen clínico de ingreso reveló un conteo inflamatorio de seis y doloroso de 14, DAS28 de 4.69, con nódulos de Bouchard y pérdida irreversible del arco del movimiento principalmente en codos y rodillas. Los exámenes de laboratorio revelaron reactantes de fase aguda elevados. La radiografía de manos mostró disminución del espacio articular en interfalángicas proximales, erosiones yuxtaarticulares, colapso del carpo. En la radiografía comparativa de rodillas se observó notorios cambios de osteoartritis con la presencia de depósitos calcáreos de varios tamaños en forma grumosa periarticular compatibles con calcinosis tumoral (Figura 1).

Se ajustó el tratamiento con DMARDS de la siguiente manera: metotrexate $17.5 \mathrm{mg} / \mathrm{s}$, sulfasalazina $2 \mathrm{~g} / \mathrm{d}$, cloroquina $250 \mathrm{mg} / \mathrm{d}$, prednisona $5 \mathrm{mg} / \mathrm{d}$, calcio $600 \mathrm{mg} / \mathrm{d}$, calcitriol $0.25 \mathrm{mg} / \mathrm{d}$, acido fólico $1 \mathrm{mg} / \mathrm{d}$. Con adecuada evolución durante tres meses de seguimiento, disminuyendo el DAS28 a 2.1 y los paraclínicos incluyendo calcio, fósforo, PTH estaban dentro de límites normales. En la actualidad la paciente no ha referido un tratamiento específico para la calcinosis tumoral y se monitoriza en cada control para buscar complicaciones derivadas de la misma (Figura 1). 


\section{Discusión}

La calcinosis tumoral (CT) también conocido como síndrome de Teutschlander, es una enfermedad benigna y rara caracterizada por masas calcificadas que se ubican en los tejidos blandos periarticulares (1). Aproximadamente un tercio de los casos tienen componente familiar y un $80 \%$ de los pacientes son negros (2). Los sitios mas afectados son la cadera, hombro, codo y en menor frecuencia pies, tobillos y rodillas (3).

La fisiopatología aún es desconocida, Smack y colaboradores (4) formularon una clasificación basada en la patogénesis: 1.CT normofosfatémica primaria, 2. CT hiperfosfatémica primaria y 3 . CT secundaria como la falla renal con hiperparatiroidismo, hipervitaminosis $\mathrm{D}$, síndrome de leche alcalina, sarcoidosis, osteolisis masiva. Se piensa que hay un defecto en la célula tubular proximal, con reabsorción elevada de fosfato e incremento en la producción de 1,25 dihidroxivitamina $\mathrm{D}$, lo que lleva a depósito de cristales de hidroxiapatita en las bursas, médula, pulpa dental, vasos sanguíneos y piel. Recientemente se ha descubierto algunas mutaciones autosómicas recesivas en los genes GALNT3 y FGF23 (5).

La manifestación clínica habitual es la formación de una masa en tejidos blandos alrededor de las articulaciones usualmente asintomática, la cual puede aumentar de tamaño, puede aparecer luego de un trauma local y rara vez ocasiona dolor articular o limitación funcional. Otras manifestaciones pueden ser debidas a la compresión nerviosa, ulceraciones cutáneas y la formación de tractos con drenaje lechoso (6).

En la radiografía convencional puede verse una calcificación grande, de forma de nube, radioopaca con septos radiolúcidos que representan paredes fibrosas de la lesión conocidos como "alambre de pollo" (7). La TAC muestra la extensión de la lesión, también permite observar niveles líquido-líquido conocido como signo de la sedimentación (8). La RNM puede mostrar edema óseo en el hueso adyacente (alta señal en T2).

Hay un amplio diagnóstico diferencial entre los que se encuentra el sarcoma sinovial, osteosarcoma, condrosarcoma, condromatosis sinovial, tofo gotoso, enfermedad por depósito de pirofosfato, dermatomiositis, polimiositis, enfermedad mixta del tejido conectivo, esclerosis sistémica.

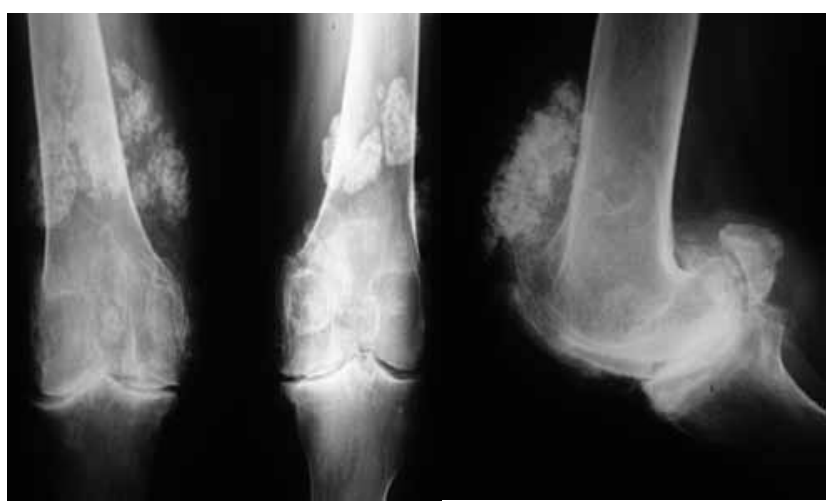

Figura 1. Rx AP y lateral de rodillas. Calcinosis tumoral manifestada por varias masas calcificadas heterogéneas en los tejidos blandos alrededor de las rodillas, también se aprecia disminución del espacio femoro-tibial y tibio-femoral con esclerosis yuxtaarticular y formación de osteofitos correspondiente a una osteoartritis.

Varios métodos han sido propuestos para el tratamiento de CT. Mozzafarian y colaboradores reportaron regresión completa bajo terapia con antiácidos en combinación con dieta baja de calcio y fósforo, aunque puede haber recurrencias (9). Seyahi y colegas reportaron la efectividad de vinpocetina en eliminar CT sin recurrencias locales (10). La escisión quirúrgica es el método recomendado para tratar esta enfermedad, pero también las recidivas son frecuentes.

\section{Referencias}

1. Mohamed S, Jong-Hung J, Weon-Yoo K. Tumoral calcinosis of the foot with unusual presentation in an 11 years old boy: a case report and review of literature. J Postgrad Med 2007; 53: 247-9.

2. Aghaghazvini L, Shirani S. Photoclinic. Arch Iranian Med 2008; 11: 227-8.

3. Prahinski J, Schaefer R. Tumoral calcicosis of the foot. Foot Ankle Int 2001; 22: $911-3$.

4. Smack D, Norton S, Fitzpatrick J. Proposal for a pathogenesis-based classification of tumoral calcinosis. Int J Dermatol 1996; 35: 265-71.

5. Olsen K, Chew F. Tumoral calcinosis: Pearls, polemics, and alternative possibilities. Radiographics 2006; 26: 871-85.

6. Martinez S, Vogler J, Harrelson J, Lyles K. Imaging of tumoral calcinosis: new observations. Radiology 1990, 174: 215-22.

7. Steinbach L, Johnson J. Tumoral calcinosis: radiologic-pathologic correlation Skeletal Radiol 1995; 24: 573-8.

8. Mockel G, Buttgereit F, Labs K, Perka C. Tumoral calcinosis revisited: pathophysiology and treatment. Rheumatol Int 2005; 25: 55-9.

9. Mozzafarin G, Lafferty F, Pearson O. Treatment of tumoral calcinosis with phosphorus deprivation. Ann Int Med 1972; 77: 741-5.

10. Seyahi A, Atalar A, Ergin H. Tumoral calcicosis: clinical and biochemical aspects of a patient treated with vinpocetine. Eur J Intern Med 2006; 17: 436-8. 